\title{
Experimental demonstration of water based tunable metasurface
}

Odit, Mikhail; Kapitanova, Polina; Andryieuski, Andrei; Belov, Pavel; Lavrinenko, Andrei V.

Published in:

Applied Physics Letters

Link to article, DOI:

$10.1063 / 1.4955272$

Publication date:

2016

Document Version

Publisher's PDF, also known as Version of record

Link back to DTU Orbit

Citation (APA):

Odit, M., Kapitanova, P., Andryieuski, A., Belov, P., \& Lavrinenko, A. V. (2016). Experimental demonstration of water based tunable metasurface. Applied Physics Letters, 109(1), [011901]. https://doi.org/10.1063/1.4955272

\section{General rights}

Copyright and moral rights for the publications made accessible in the public portal are retained by the authors and/or other copyright owners and it is a condition of accessing publications that users recognise and abide by the legal requirements associated with these rights.

- Users may download and print one copy of any publication from the public portal for the purpose of private study or research.

- You may not further distribute the material or use it for any profit-making activity or commercial gain

- You may freely distribute the URL identifying the publication in the public portal 


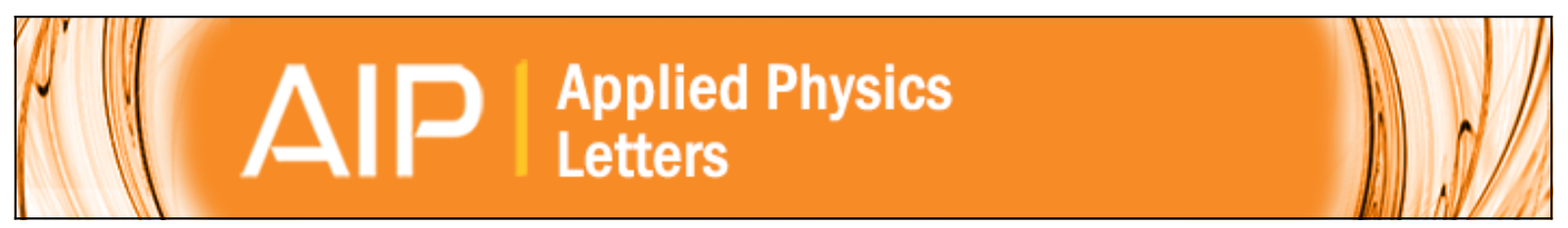

\section{Experimental demonstration of water based tunable metasurface}

Mikhail Odit, Polina Kapitanova, Andrei Andryieuski, Pavel Belov, and Andrei V. Lavrinenko

Citation: Applied Physics Letters 109, 011901 (2016); doi: 10.1063/1.4955272

View online: http://dx.doi.org/10.1063/1.4955272

View Table of Contents: http://scitation.aip.org/content/aip/journal/apl/109/1?ver=pdfcov

Published by the AIP Publishing

\section{Articles you may be interested in}

Ultra-wideband and high-efficiency polarization rotator based on metasurface

Appl. Phys. Lett. 109, 051901 (2016); 10.1063/1.4960355

Symmetry-based coding method and synthesis topology optimization design of ultra-wideband polarization conversion metasurfaces

Appl. Phys. Lett. 109, 014104 (2016); 10.1063/1.4955412

Coherent perfect absorption in an all-dielectric metasurface

Appl. Phys. Lett. 108, 121901 (2016); 10.1063/1.4944635

Fe-based ferromagnetic microwires enabled meta-composites

Appl. Phys. Lett. 103, 251902 (2013); 10.1063/1.4850196

Ultra-broadband electromagnetically induced transparency using tunable self-asymmetric planar metamaterials J. Appl. Phys. 114, 163507 (2013); 10.1063/1.4826630

\section{Precise temperature control for cryogenic research}

\section{Model 372}

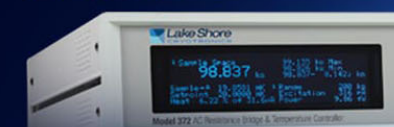




\title{
Experimental demonstration of water based tunable metasurface
}

\author{
Mikhail Odit, ${ }^{1}$ Polina Kapitanova, ${ }^{1}$ Andrei Andryieuski, ${ }^{2}$ Pavel Belov, ${ }^{1}$ \\ and Andrei V. Lavrinenko ${ }^{2}$ \\ ${ }^{1}$ Department of Nanophotonics and Metamaterials, ITMO University, St. Petersburg 197101, Russia \\ ${ }^{2}$ DTU Fotonik, Technical University of Denmark, Ørsteds Plads, Bld. 343, DK-2800 Kgs. Lyngby, Denmark
}

(Received 11 April 2016; accepted 23 June 2016; published online 5 July 2016)

\begin{abstract}
A simple dynamically tunable metasurface (two-dimensional metamaterial) operating at microwave frequencies is developed and experimentally investigated. Conceptually, the simplicity of the approach is granted by reconfigurable properties of unit cells partially filled with distilled water. The transmission spectra of the metasurface for linear and circular polarizations of the incident wave were experimentally measured under the metasurface rotation around a horizontal axis. The changes in the transmission coefficient magnitude up to $8 \mathrm{~dB}$ at $1.25 \mathrm{GHz}$ are reported while rotating the metasurface by the $90^{\circ}$ angle. The proposed approach manifests the cheap and accessible route for the electromagnetic wave control in the microwave region with the help of metasurfaces. Published by AIP Publishing. [http://dx.doi.org/10.1063/1.4955272]
\end{abstract}

The scientific field of metamaterials has opened wide opportunities to access by artificial structuring electromagnetic properties not available in nature. Among different classes of metamaterials, the all-dielectric metamaterials ${ }^{1}$ (and their twodimensional analogues - metasurfaces ${ }^{1-3}$ ) are currently considered to have great potential towards low-loss alternatives to intrinsically lossy metal-based designs. The functionality of such all-dielectric structures is typically based on high values of a dielectric permittivity, which serve to compensate the lack of free carriers by employing Mie resonances.

In microwaves, various high-dielectric materials with permittivities on the order of several hundreds (see, for example, Vendik et $a l .^{8}$ ) were tested for such purposes. In most cases that implies using relatively expensive materials, like, for example, barium-strontium titanate. However, in order to reach massive practical applications, the metamaterials must be cost-efficient, technologically flexible, and, desirably, ecologically friendly. If in addition the metamaterial enables dynamic tuning of its properties, it will very likely become a working horse of metasurfaces/metamaterials designing.

A few tunable configurations have been reported so far mostly in the optical range: temperature tuning of a silicon nanodisks metasurface filled with a liquid crystal; ${ }^{4}$ mechanically tunable metasurface with titania nanodisks; ${ }^{5}$ carrier concentration tuning of Mie resonances in $\mathrm{Si}$ or Ge nanoparticles; ${ }^{6}$ and transmission modulation of a Si metasurface loaded with a graphene layer. ${ }^{7}$

Surprisingly enough, but one of the most abundant, easily reconfigurable and by default nature-friendly material on Earth, namely, water has been considered as a material platform for microwave metamaterials only very recently. ${ }^{9-11}$ In this frequency range water has high dielectric permittivity $\varepsilon$, which in addition heavily depends on temperature $T .{ }^{12}$ In the normal laboratory conditions, water is a liquid in the large temperature range between 0 and $100^{\circ} \mathrm{C}$. As such, it preserves its volume, but takes the shape provided by a container, which minimizes its potential energy (thus the surface of water in rest is flat besides the wetting effect).
So, the synergy of these two basic properties of waterthe high permittivity and easy volume reshaping-manifests the remarkable conditions for designing microwave tunable metamaterials with advanced functionalities. Andryieuski et $a l .{ }^{9}$ proposed to use the properties of water for tunable transmission of electromagnetic waves through a system of partially filled water meta-atoms. Tunability is provided by the gravity force, which readily reshapes the water volume in each elementary reservoir with rotation of the whole system of the meta-atoms. In such configuration, the water-based metamaterials can be definitely considered as the simplest and potentially the cheapest realization of a tunable metamaterial or metasurface. In this Letter, we provide the experimental demonstration of this functional device.

The metasurface (two-dimensional metamaterial) under study is composed of unit cells periodically arranged in a square lattice with period $d=6 \mathrm{~cm}$ [Fig. 1(a)]. An individual unit cell consists of an empty elliptical cylinder partially filled with water. The elliptical cylinder has the following parameters: major axis $a=2.5 \mathrm{~cm}$, minor axis $b=1 \mathrm{~cm}$, and height (or thickness) $h=1 \mathrm{~cm}$. In principle, tapped water can be used as the filling of the elliptical cylinders. In our case, we employed commercially available distilled water. The distilled water permittivity was experimentally studied in the microwave frequency range of $1-2 \mathrm{GHz}$ at room temperature
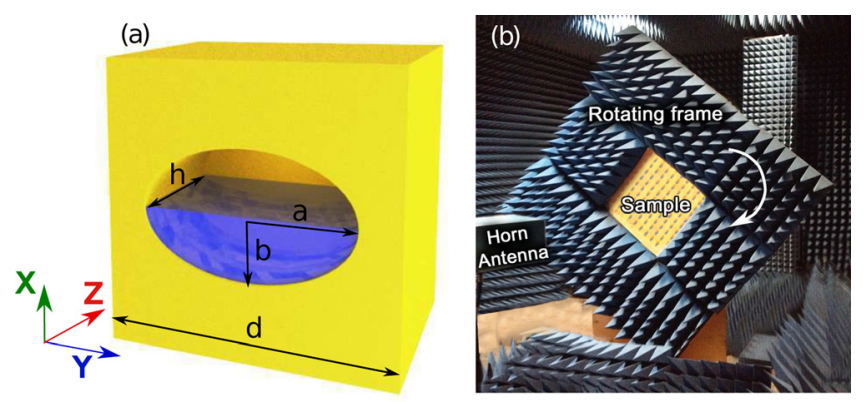

FIG. 1. (a) Schematic of the unit cell partially filled with water. (b) Photograph of the experimental setup. The metasurface is in the middle of the reflectionless frame. 
$T=24{ }^{\circ} \mathrm{C}$ using the Rohde\&Schwarz ZVB 20 Vector Network Analyzer (VNA) and a probe of the dielectric assessment kit (DAK-3.5). ${ }^{13}$ The measured data correspond well to the data known from literature, ${ }^{12}$ for example, at $1.25 \mathrm{GHz} \epsilon=78+\mathrm{i} 4.7$. The dimensions of the elliptical cylinders were specifically selected to access the Mie resonances in the frequency range of $1.2-2 \mathrm{GHz}$. The array of such unit cells can be considered to operate in the metamaterial regime rather than as a diffraction grating. The metasurface rotation angle is set to $0^{\circ}$, when the major axis of the elliptical cylinder $a$ is parallel to the $y$-axis [see Fig. 1(a)]. With the metasurface clockwise rotation up to $90^{\circ}$, the water flows and changes its shape as illustrated in the inset of Fig. 2(c). In this manner, the metasurface is dynamically tunable with its transmission depending on the rotation angle.

Full-wave modeling of the metasurface transmission coefficient upon the plane-wave excitation was performed using the frequency domain CST Microwave Studio solver. Two Bloch-Floquet ports located in the far-field of the unit cell along the $z$ direction were used. The unit cell periodic boundary conditions were imposed in simulations, so that the structure and the incident wave are periodically repeated along the elliptical cylinder major axis. To demonstrate the tunability, we specifically characterized the metasurface transmission in dependence on rotation around the $z$-axis. We considered the following types of polarization of the plane wave propagating in the $z$ direction: (i) the horizontal polarization with the electric field polarized along the $y$-axis $\mathrm{E} \| \mathrm{y}$ (at the zero rotation angle, the major axis $a$ is parallel to the electric field) [see Fig. 1(a)]; (ii) the vertical polarization with the electric field polarized along the $x$-axis $\mathrm{E} \| \mathrm{x}$ (at the zero rotation angle the minor axis $b$ is parallel to the electric field); and (iii) right (RCP) and left (LCP) circular polarizations.

First, we numerically studied the metasurface transmission properties for a horizontally polarized plane wave. The results obtained for different rotation angles are depicted in Fig. 2(a). As one can see under the $0^{\circ}$ rotation angle, the minimum of the transmission coefficient magnitude is 0.35 at the frequency near $1.2 \mathrm{GHz}$. Here and in the rest of the paper discussing about the transmission coefficient we refer to its magnitude. Rotation of the metasurface increases the transmission coefficient up to 0.95 .
To verify the effect, the prototype of the tunable waterfilled metasurface was fabricated and experimentally investigated in an anechoic chamber [Fig. 1(b)]. The array of $9 \times 9$ elliptical cylinders was drilled in a custom holder made of a Styrofoam material with a permittivity close to 1 at the microwave frequencies. All elliptical cylinders were halffilled by injection of $12 \mathrm{ml}$ of distilled water. The metasurface was fixed in the specially designed rotating frame. In order to reduce the edge diffraction and its influence on the transmission characteristics, the metasurface perimeter was surrounded by microwave absorbers [Fig. 1(b)]. To perform a plane wave excitation and detection, a pair of identical rectangular linearly polarized broadband horn antennas (operational range of $1-18 \mathrm{GHz}$ ) connected to the coaxial ports of an Agilent E8362C Vector Network Analyzer was used. The metasurface was located at the distance of $2 \mathrm{~m}$ to both the transmitting and receiving antennas. In the case of a circularly polarized wave, the horn antennas were replaced by a self-made narrow-band right circular polarized antennas. ${ }^{14}$ The antennas were matched better than $-10 \mathrm{~dB}$ in the frequency band of 1-2 GHz.

During the characterization, the complex transmission coefficient of the metasurface was measured for rotation angles in the range of $0^{\circ}-90^{\circ}$. The background signal was subtracted by means of the free space measurement. ${ }^{15}$ In order to minimize the effect of the additional reflections from the walls of the anechoic chamber, the measured signal was transformed into the time domain with the subsequent time-domain gating. ${ }^{16}$

The measured transmission coefficients of the metasurface in the case of the horizontal polarization of the excitation wave are shown in Fig. 2(b). We observe good agreement between the simulated results and measured data. The magnitudes of the measured and simulated transmission coefficients as a function of the rotation angle are directly compared in Fig. 2(c). Following the positions of the minimal transmission, we picked up the frequency $1.2 \mathrm{GHz}$ for presentation of the simulated results (green solid line) and $1.25 \mathrm{GHz}$ for presentation of the measured ones (green dots). The little discrepancy in the resonance frequency can be attributed mainly to the tolerance in the custom holder fabrication and deviation of the metasurface orientation relative to the incident plane wave. One can see that the dynamical
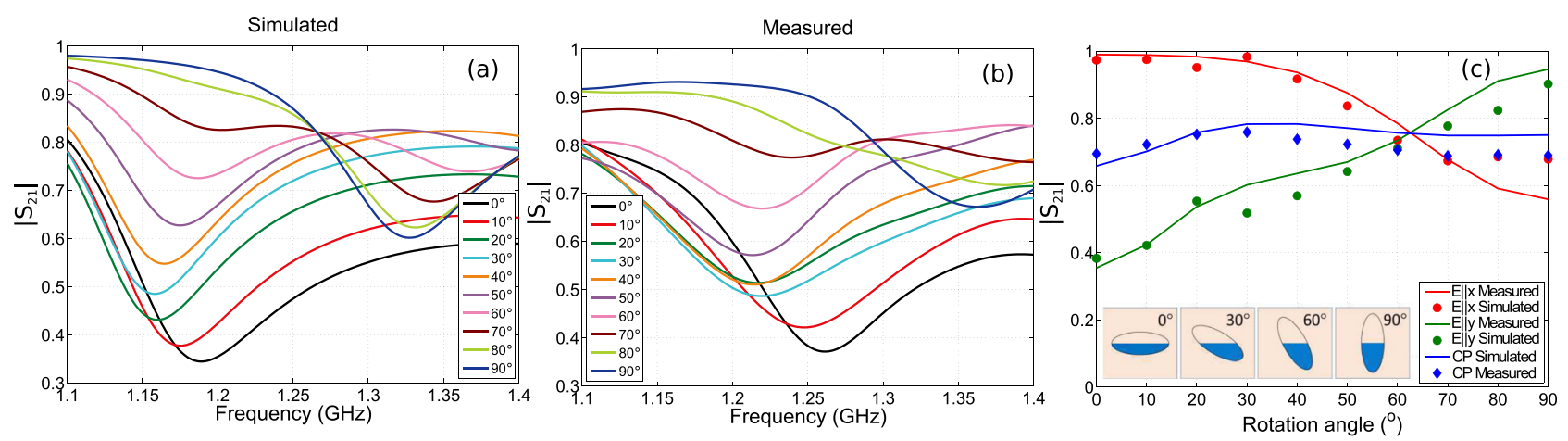

FIG. 2. Simulated (a) and measured (b) transmission coefficient magnitudes for horizontally polarized incident plane-wave at different rotation angles (from $0^{\circ}$ to $90^{\circ}$ ). (c) Comparison of the simulated (solid lines) at $\mathrm{f}=1.2 \mathrm{GHz}$ and measured (dots) at $\mathrm{f}=1.25 \mathrm{GHz}$ transmission coefficient magnitudes as a function of the rotation angle for horizontal, vertical, and right circular polarizations of the incident wave. The inset shows the unit cell rotation on different angles and corresponding water cell shape. Here, CP denotes to RCP-RCP polarizations. 
range of variation in the transmission coefficient magnitude by rotating the metasurface from $0^{\circ}$ to $90^{\circ}$ is extended from 0.38 to 0.90 .

Next, we studied both numerically and experimentally the tunability of the water based metasurface under the vertically polarized plane wave excitation. The results of simulations are depicted in Fig. 3(a). In contrast to the case of the horizontal polarization, the transmission coefficient is almost constant in the frequency band $1.1-1.4 \mathrm{GHz}$ under the $0^{\circ}$ rotation angle. With the rotation angle increasing up to $90^{\circ}$, the minimum in the magnitude builds up near the frequency 1.2 GHz. The measured transmission coefficient magnitude is shown in Fig. 3(b). Again besides the same minimum position shift from 1.2 to $1.25 \mathrm{GHz}$ as for the horizontal polarization case the measured data agree well with the simulated results. The magnitudes of the measured and simulated transmission coefficients as a function of the rotation angle are compared in Fig. 2(c). The simulated results at frequency 1.2 $\mathrm{GHz}$ are shown by the red solid line whereas the measured data at frequency $1.25 \mathrm{GHz}$ are depicted by the red dots. For the vertical polarization measured magnitude of transmission coefficient changes from 0.97 to 0.67 by rotating the metasurface by $90^{\circ}$ (while simulated data changes from 0.99 to $0.55)$.

Finally, modeling and characterization of the metasurface excited by a circular polarized wave were performed. Such experiments can clearly demonstrate the effect of water

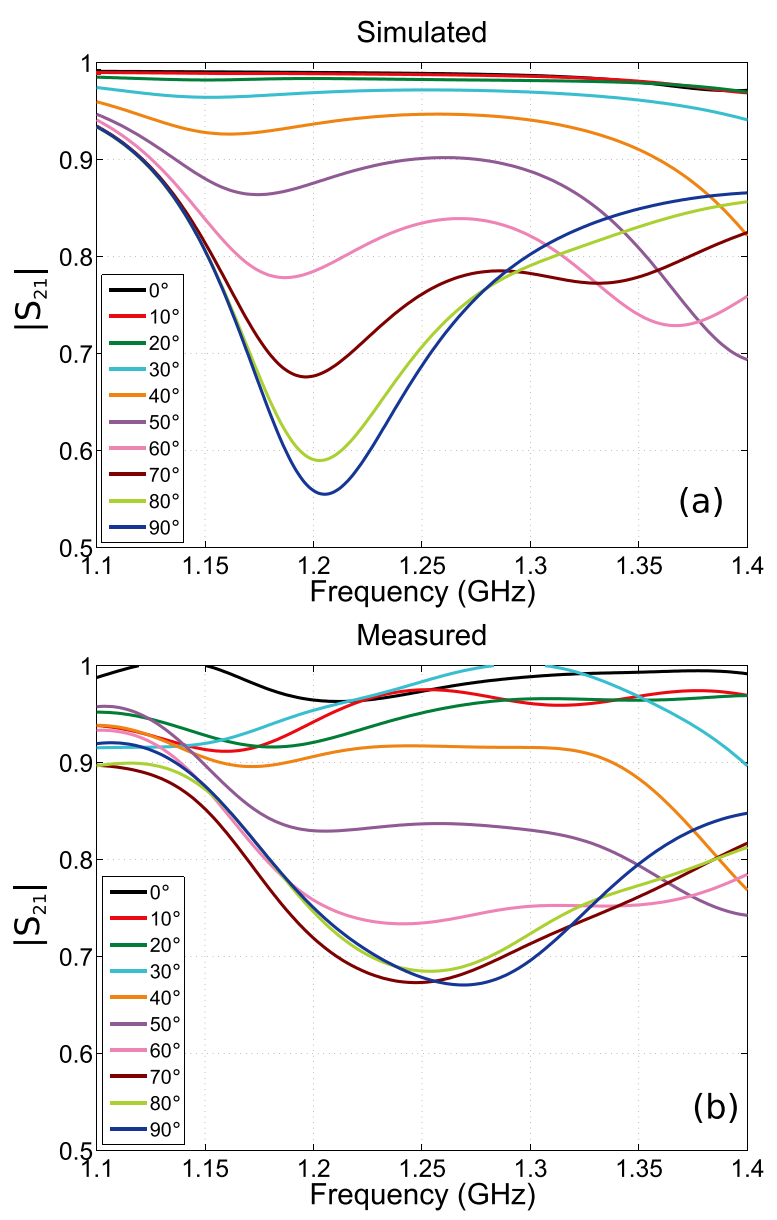

FIG. 3. Simulated (a) and measured (b) transmission coefficient magnitude for the vertically polarized excitation plane wave at different rotation angles. redistribution in the unit cells. Indeed, if all unit cells are fully filled with water, rotating of the metamaterial should have no effect on the circular polarized wave transmission. We modeled the RCP-RCP transmission as well as the polarization conversion from RCP to LCP. The results of numerical simulations are shown in Fig. 4(a): the RCP-RCP transmission by the solid lines and RCP-LCP conversion by the dashed lines. One can conclude that the transmission coefficient magnitudes have dependence on the angle of rotation for circular polarized waves although less pronounced than for linear polarized waves. For example, at the frequency in the vicinity of $1.15 \mathrm{GHz}, \mathrm{RCP}-\mathrm{LCP}$ conversion drops down while RCP-RCP transmission coefficient increases with the angle of rotation. Such behavior towards circular polarization can be explained by the bianisotropy of the metasurface made of partially filled elliptical cylinders; however, detailed characterization of the bianisotropic properties of the metasurface is beyond the scope of the Letter.

Two RCP-antennas and one LCP-antenna were fabricated in order to perform circular polarization characterization. Antennas were matched in the frequency range of $1-2$ GHz. The results of transmission coefficient measurements (Figs. 4(b)) agree very well with the modeling results.

In conclusion, we have assembled and characterized extremely simple and cheap metasurface for the microwave frequency range, which is easily tunable just by adjusting of
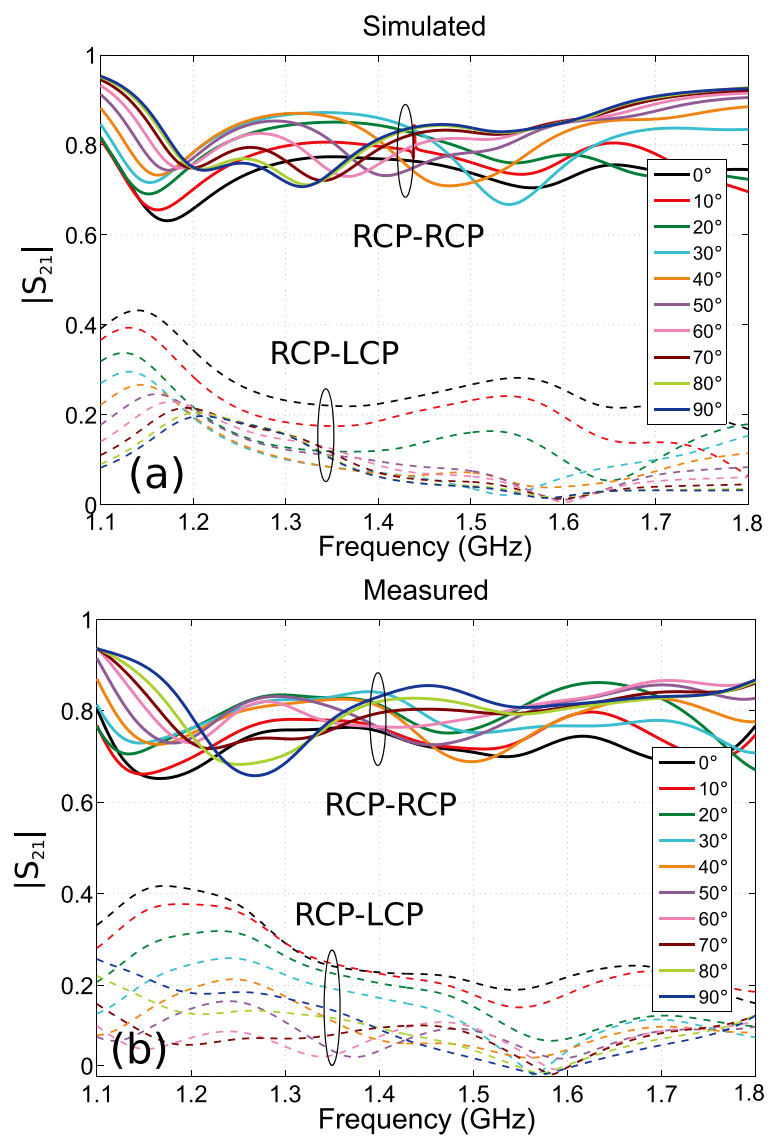

FIG. 4. Simulated (a) and measured (b) transmission coefficient magnitudes of the metasurface for the circular polarization of the incident wave. The solid lines demonstrate the transmission coefficient for the right circularly polarized wave accepted by the right circularly polarized antenna. The dashed lines demonstrate the transmission coefficient for the right circularly polarized wave accepted by the left circularly polarized antenna. 
the rotation angle. The metasurface is composed of elliptical cylinders partially filled with water. We have confirmed the very principle of the metasurface controllable operation in direct experiments, namely, that by rotation of the whole array of cylinders transmission properties of the metasurface can be easily altered due to the reshaping of the water metaatoms in the elliptical voids. The demonstrated range of transmission variation is relatively modest, between 0.4 and 1.0 in magnitude (from $-8 \mathrm{~dB}$ to $0 \mathrm{~dB}$ ), but it can be well expanded by considering, for example, other shapes of the water inclusions with larger asymmetry. Although it has not been the subject of this particular study, additional tunability can be implemented by means of water temperature control. We believe that the proposed architecture of tunable metasurfaces can find a wide application in microwave technology, especially for demonstration and cost efficient prototyping of functional artificial electromagnetic surfaces.

The numerical simulation of the metasurface was supported by the Russian Foundation for Basic Research (Project No. 15-32-20665). The experimental investigation of the metasurface performance was supported by Russian Science Foundation (Project 14-12-00897). P.K. acknowledges the scholarship of the President of Russian Federation. The authors acknowledge partial support from the Villum Fonden and are thankful to D. Dobrykh for circularly polarized antennas fabrication.

${ }^{1}$ S. Jahani and Z. Jacob, “All-dielectric metamaterials," Nat. Nanotechnol. 11, 23-36 (2016).

${ }^{2}$ L. Shi, T. U. Tuzer, R. Fenollosa, and F. Meseguer, "A new dielectric metamaterial building block with a strong magnetic response in the sub1.5-micrometer region: Silicon colloid nanocavities," Adv. Mater. 24, 5934-5938 (2012).
${ }^{3}$ Y. F. Yu, A. Y. Zhu, R. Paniagua-Dominguez, Y. H. Fu, B. Luk'yanchuk, and A. I. Kuznetsov, "High-transmission dielectric metasurface with $2 \pi$ phase control at visible wavelengths," Laser Photonics Rev. 9, 412-418 (2015).

${ }^{4}$ J. Sautter, I. Staude, M. Decker, E. Rusak, D. N. Neshev, I. Brener, and Y. S. Kivshar, "Active tuning of all-dielectric metasurfaces," ACS Nano 9, 4308-4315 (2015).

${ }^{5}$ P. Gutruf, C. Zou, W. Withayachumnankul, M. Bhaskaran, S. Sriram, and C. Fumeaux, "Mechanically tunable dielectric resonator metasurfaces at visible frequencies," ACS Nano 10, 133-141 (2016).

${ }^{6}$ T. Lewi, P. P. Iyer, N. A. Butakov, A. A. Mikhailovsky, and J. A. Schuller, "Widely tunable infrared antennas using free carrier refraction," Nano Lett. 15, 8188-8193 (2015).

${ }^{7}$ C. Argyropoulos, "Enhanced transmission modulation based on dielectric metasurfaces loaded with graphene," Opt. Express 23, 23787-23797 (2015).

${ }^{8}$ I. Vendik, M. Odit, and D. Kozlov, "3D metamaterial based on a regular array of resonant dielectric inclusions," Radioengineering 18, 111-116 (2009).

${ }^{9}$ A. Andryieuski, S. M. Kuznetsova, S. V. Zhukovsky, Y. S. Kivshar, and A. V. Lavrinenko, "Water: promising opportunities for tunable alldielectric electromagnetic metamaterials," Sci. Rep. 5, 13535 (2015).

${ }^{10}$ M. V. Rybin, D. S. Filonov, K. B. Samusev, P. A. Belov, Y. S. Kivshar, and M. F. Limonov, "Phase diagram for the transition from photonic crystals to dielectric metamaterials," Nat. Commun. 6, 10102 (2015).

${ }^{11}$ Y. J. Yoo, S. Ju, S. Y. Park, Y. J. Kim, J. Bong, T. Lim, K. W. Kim, J. Y. Rhee, and Y. P. Lee, "Metamaterial absorber for electromagnetic waves in periodic water droplets," Sci. Rep. 5, 14018 (2015).

${ }^{12} \mathrm{~W}$. J. Ellison, "Permittivity of pure water, at standard atmospheric pressure, over the frequency range $0-25 \mathrm{THz}$ and the temperature range $0-100{ }^{\circ} \mathrm{C}$, , Phys. Chem. Ref. Data 36, 1-18 (2007).

${ }^{13}$ See http://www.speag.com/products/dak/dielectric-measurements/ for the description of Dielectric Assessment Kit specification and features.

${ }^{14} \mathrm{~V}$. Wongpaibool, Experiment on triangular copper-strip as impedance matching for 2.4-GHz helical antenna. Assumption Univ. J. Technol. 11(4), 209-214 (2008).

${ }^{15}$ C. Larsson, C. Sohl, M. Gustafsson, and G. Kristensson, "Extinction cross section measurements," in Proceedings of Nordic Conference on Radio Science and Communications (2008), pp. 127-129.

${ }^{16}$ R. De Porrata-Doria, A. B. Ibars, and L. F. Martinez, "Analysis and reduction of the distortions induced by time-domain filtering techniques in network analyzers,” IEEE Trans. Instrum. Meas. 47, 930-934 (1998). 